\title{
STABILITY OF SEMILINEAR STOCHASTIC EVOLUTION EQUATIONS WITH MONOTONE NONLINEARITY
}

\author{
RUHOLLAH JAHANIPUR AND BIJAN Z. ZANGENEH
}

\begin{abstract}
In this paper, we consider the exponentially asymptotic stability of the mild solutions of semilinear stochastic evolution equations of monotone type. An Itô-type inequality is our main tool to study the stability in the $p$-th moment and almost sure sample-path stability of the mild solutions. We also give some examples to illustrate the applications of the theorems.
\end{abstract}

\section{Mathematics subject classification (1991): 60H15, 34G20.}

Key words and phrases: Stochastic evolution equations, Itô-type inequality, Stability in the $p$-th moment, Borel-Cantelli's lemma, Sample-path stability.

\section{REFERENCES}

[1] BRowder, F. E., Non-linear equations of evolution. Ann. of Math., 80(1964), 485-523.

[2] Caraballo, T. \& Real, J., On the pathwise exponential stability of nonlinear stochastic partial differential equations. Stochastic Anal. Appl., 12(1994), 517-525.

[3] Caraballo, T., \& LiU, K., Exponential stability of mild solutions of stochastic partial differential equations with delays. Stochastic Anal. Appl., 17(1999), 743-763.

[4] CuRTain, R. F., Stochastic evolution equations with general white noise disturbance. J. Math. Anal. Appl., 60(1977), 570-595.

[5] Curtain, R. F. \& PRitchard, A. J., Infinite dimensional linear system theory. LN in control and information sciences (8), Springer-Verlag, Berlin-Heidelberg, New York, 1978.

[6] DA Prato, G. \& ZABCZYK, J., Stochastic equations in infinite dimensions. Cambridge, 1992.

[7] Haussmann, U. G., Asymptotic stability of the linear Itô equation in infinite dimentions. J. Math. Anal. Appl., 65 (1978), 219-235.

[8] ICHIKAWA, A., Dynamic programming approach to stochastic evolution equations. SIAM J. Control Optim., 17(1979), 152-174.

[9] ICHIKAWA, A., Stability of semilinear stochastic evolution equations. J. Math. Anal. Appl., 90(1982), $12-44$.

[10] ICHIKAWA, A., Semilinear stochastic evolution equations: boundedness, stability and invariant measures. Stochastics, 12(1984), 1-39.

[11] KHAS'MINSKII, R., Stochastic stability of differential equations. Sijthoff \& Noordhoff, Netherlands, 1980.

[12] Kotelenez, P., A submartingale type inequality with applications to stochastic evolution equations. Stochastics, 8(1982), 139-151.

[13] KoteleneZ, P., A stopped Doob inequality for stochastic convolution integrals and stochastic evolution equations. Stochastic Anal. Appl., 2(1984), 245-265.

[14] Leha, G. \& Maslowski, B. \& Ritter, G., Stability of solutions to stochastic evolution equations. Stochastic Anal. Appl., 17(1999), 1009-1051.

[15] LIU, K., Lyapunov functionals and asymptotic stability of stochastic delay evolution equations. Stochastics, 63(1998), 1-26.

[16] LiU, K. \& MAO, X., Exponential stability of non-linear stochastic evolution equations. Stochastic Process. Appl., 78(1998), 173-193. 
[17] LiU, R. \& MANDREKAR, V., Stochastic semilinear evolution equations: Liapunov function, stability and ultimate boundedness. J. Math. Anal. Appl., 212(1997), 537-553.

[18] MANDREKAR, V., On Lyapunov stability theorems for stochastic (deterministic) evolution equations. Stoch. Anal. Appl. in Physics, 219-237, Kluwer Acad. Publ., Dordrecht, 1994.

[19] MAO, X., Stochastic differential equations\& applications. Horwood Publishing, 1997.

[20] MÉTIVIER, M., Semimartingales : a course on stochastic processes. Walter de Gruyter, Berlin, New York, 1982.

[21] PardouX, E., Intégrales stochastiques Hilbertiennes. Cahier de Mathématiques de la Décision(7617). Université Paris-Dauphiné, 1976.

[22] PAZY, A., Semigroups of linear operators and applications to partial differential equations. Applied Mathematical Sciences (44), Springer-Verlag, Berlin, 1983.

[23] Tanabe, H., Equations of evolution. Pitman, London, 1979.

[24] TANIGUCHI, T., Asymptotic stability theorems of semilinear stochastic evolution equations in Hilbert spaces. Stochastics, 53(1995), 41-52.

[25] YoR, M., Existence et unicité de diffusions à valeurs dans un espace de Hilbert. Ann. Inst. H.Poincaré, 10(1974), 55-88.

[26] ZABCZYK, J., On the stability of infinite dimensional linear stochastic systems. Probability Theorey, Banach Center Publications, vol. 5(1979), 273-281.

[27] Z.ZAngeneH, B., Semilinear stochastic evolution equations. Ph.D thesis, University of British Columbia, Vancouver, B.C. Canada, 1990.

[28] Z. ZANGENEH, B., Semilinear stochastic evolution equations with monotone non-linearities. Stochastics, 53(1995), 129-174.

[29] Z. ZANGeneH, B., An energy-type inequality. Math. Ineq. Appl., 1 (1998), 453-461. 